\title{
Économie sociale, innovation et développement local : l'exemple de la CUMA
}

\author{
Carol Saucier \\ Université du Québec à Rimouski
}

\section{Introduction}

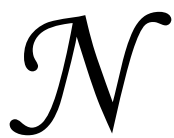
ous voulons parler ici d'un type de coopérative de producteurs apparu récemment dans le paysage québécois de la coopération. Il s'agit de la Coopérative d'utilisation de matériel agricole (CUMA). Nous chercherons, en décrivant les caractéristiques des pratiques de ces coopérateurs cumistes, à faire le lien avec le développement local et, de plus, à établir un parallèle entre cette expérimentation sociale et l'innovation.

La coopération agricole ne date pas d'aujourd'hui au Québec. Le secteur des coopératives agricoles constitue encore un des vecteurs stratégiques du coopératisme; pensons par exemple à des coopératives telles que Agropur, Purdel, la Coop fédérée (anciennement la Coopérative fédérée de Québec) œuvrant dans la transformation de produits laitiers, ovins et porcins, et dans leur mise en marché. Les coopératives d'utilisation de matériel agricole sont cependant assez récentes, les premières étant apparues dans les années 1990 et précisément dans la région du Bas-SaintLaurent. La CUMA est une formule par laquelle
Les trois composantes principales de I'univers de l'économie sociale sont les coopératives, les mutuelles et les associations à vocation économique. teurs. Pour y arriver, ils ont dû apprendre à utiliser autrement, de manière plus solidaire, le matériel agricole. Ceci les amènera à créer de nouveaux liens entre eux, à modifier leurs relations sociales. En cela, ce sont des agents innovateurs. Non pas que la formule soit nouvelle. En effet, les premières CUMA se sont développées en France dans le contexte d'aprèsguerre des années 1950 : pénurie de machinerie, coût prohibitif de celle-ci en achat individuel, contexte de reconstruction économique nationale. Les cumistes au Québec évoluent, eux, dans un contexte de fragilisation de leurs collectivités rurales d'appartenance, dans un environnement marqué par le libre-échange et la globalisation des marchés. Ils vont importer et adapter cette formule coopérative dans un nouveau contexte socio-historique. Si la formule n'est pas nouvelle, son application s'adaptera au contexte québécois des années 1990-2000.

Le présent article comprend deux parties et une conclusion. La première mettra en correspondance les concepts d'économie sociale, d'innovation et de développement local. La seconde partie décrira les pratiques des producteursmembres des CUMA des producteurs agricoles se mettent en commun pour former une coopérative, celle-ci devenant propriétaire collectif du matériel que loueront les producteursmembres. En 2004, on retrouve 64 CUMA au Québec réparties dans de nombreuses régions. Les activités déployées par les CUMA auront des effets positifs sur le développement des collectivités rurales dans lesquelles elles se sont enracinées.

Plus largement, nous ferons état du lien existant entre ces pratiques coopératives et l'innovation sociale. Les cumistes ont répondu à leur besoin de réduire substantiellement l'endettement individuel des agricul- comme illustration de cette convergence. Enfin, la conclusion permettra de synthétiser les principaux effets des CUMA sur leurs collectivités territoriales.

\section{Économie sociale, innovation et développement local}

Les trois composantes principales de l'univers de l'économie sociale sont les coopératives, les mutuelles et les associations à vocation économique. Ces organisations peuvent être regroupées également sous le vocable d'entreprises collectives. Ces dernières, nous rappelle Danielle Demoustier ${ }^{1}$, réfèrent toutes à 
des valeurs humanistes et à des principes de justice sociale. Les entreprises de l'économie sociale répondent à des besoins jugés essentiels par les acteurs qui s'y mobilisent, ces besoins étant non satisfaits ou mal satisfaits par le marché ou par l'État. En plus de répondre à des besoins économiques et sociaux de leurs membres ou de leurs collectivités, plusieurs de ces entreprises poursuivent des aspirations et des objectifs plus larges tels que l'amélioration de la qualité de vie, la démocratisation de la société, le développement durable des collectivités, l'occupation du territoire. Dans le contexte contemporain du développement de l'économie sociale au Québec, il existe une définition ralliant un grand nombre d'acteurs sociaux; il s'agit de celle du Chantier de l'économie sociale. En voici les éléments ${ }^{2}$ :

«Le domaine de l'économie sociale regroupe l'ensemble des activités et organismes, issus de l'entrepreneuriat collectif, qui s'ordonnent autour des principes et règles suivants :

1) l'entreprise d'économie sociale a pour finalité de servir ses membres ou la collectivité plutôt que de simplement engendrer des profits et viser le rendement financier;

2) elle a une autonomie de gestion à l'égard de l'État;

3) elle intègre dans ses statuts et façons de faire un processus démocratique impliquant usagères et usagers, travailleurs et travailleuses;

4) elle défend la primauté des personnes et du travail sur le capital dans la répartition de ses surplus et revenus;

5) elle fonde ses activités sur le principe de la participation, de la prise en charge, de la responsabilité individuelle et collective. »

$\mathrm{Au}$ sujet de la première caractéristique, il nous apparaît important de faire la spécification suivante à l'instar de Jacques Defourny. Ainsi : «L'entreprise d'économie sociale est en elle-même, par la nature de son activité, un service rendu à ses membres ou à d'autres personnes et non un outil de rapport financier. Le profit nécessaire aux entreprises ne pouvant compter sur certaines formes de subsidiation est alors un moyen de réaliser ce service et non le mobile principal de l'activité $»^{3}$. Par exemple, dans le cas des coopératives, qui pour la plupart reçoivent peu ou pas d'aide financière publique, le profit ou le surplus recherché est un moyen de réaliser le service offert aux membres et non le motif principal de l'activité. À la troisième caractéristique, il convient d'ajouter la règle démocratique prévalant et qui est celle d'une personne égale une voix (un membre = une voix).

\section{L'innovation sociale se fait novatrice parce qu'elle représente une solution hors norme dans un contexte donné.}

Examinons maintenant le concept d'innovation sociale. Le Centre de recherche sur les innovations sociales (CRISES) en donne la définition suivante : «Une innovation sociale est une intervention initiée par des acteurs sociaux pour répondre à une aspiration, subvenir à un besoin, apporter une solution ou profiter d'une opportunité d'action afin de modifier des relations sociales, de transformer un cadre d'action ou de proposer de nouvelles orientations culturelles. »

On peut également définir l'innovation sociale en s'intéressant à ses dimensions que sont la forme, le processus de création et de mise en œuvre, les acteurs et les objectifs de changement qu'elle poursuit. Nous aborderons donc la question du quoi, du comment, du qui et du pourquoi.

La forme de l'innovation (Quoi ?) Pour certains auteurs, l'innovation sociale est immatérielle. Elle fait essentiellement référence à des "façons de faire ", des actions, des pratiques. Elle s'oppose donc à la notion de produit. Toutefois, pour d'autres, l'innovation sociale peut prendre la forme de produits tangibles Dans tous les cas, l'innovation sociale se fait novatrice parce qu'elle représente une solution hors norme dans un contexte donné.

Le processus innovateur (Comment ?) L'innovation sociale se particularise par la participation des usagers au processus et ce, de la prise de conscience du besoin à la conception du projet, jusqu'à sa mise en œuvre. L'innovation est animée par le souci de donner au groupe concerné par le projet la maîtrise de sa conception et de son déroulement.

Les acteurs innovants (Qui ?) Outre les usagers qui en constituent les protagonistes, et selon la forme 
qu'elle prend, l'innovation sociale est susceptible d'associer plusieurs types d'acteurs.

Les objectifs poursuivis (Pourquoi ?) L'innovation vise la résolution de problèmes sociaux. Par exemple : se défendre collectivement, se reconnaître, créer, améliorer ses conditions de vie. Plus précisément, elle vise à satisfaire les besoins non ou mal satisfaits par les moyens « officiels ». L'innovation peut être envisagée comme un processus d'apprentissage dont l'objectif consiste en la «valorisation individuelle » et l' « autonomisation » des usagers en vue de leur permettre de résoudre leurs problèmes.

\section{Les Coopératives d'utilisation de matériel agricole sont un phénomène relativement récent au Québec.}

Outre le fait que toutes les initiatives d'économie sociale ne constituent pas d'emblée l'expérimentation de pratiques hors norme dans un contexte donné, il existe des passerelles entre l'économie sociale et l'innovation sociale. Les acteurs d'économie sociale, et notamment les coopérateurs, développent des produits ou services afin de résoudre divers problèmes auxquels ils sont confrontés, ceci dans le but d'améliorer leurs conditions de vie, voire celles de leur milieu. La réponse satisfaisante à ces besoins ne vient ni du marché ni de l'État. En cela, ces acteurs rejoignent les motifs qui animent les agents innovateurs. De plus, la gestion démocratique est un principe fondamental des coopératives et dicte aussi des façons de faire conséquentes encourageant la responsabilisation individuelle et collective des membres. Là également, les coopérateurs peuvent être en phase avec le processus d'innovation sociale, lequel ne peut s'exercer que de manière participative impliquant complètement les protagonistes de l'innovation.

Comme nous voulons établir les convergences possibles entre économie sociale, innovation et développement, il nous reste à jeter un coup d'œil au concept de développement. Plusieurs auteurs ont souligné l'évolution des politiques de développement des années 1960-1970, politiques principalement impulsées par l'État, vers des politiques de développement plus endogènes, dites de développement local. Ces politiques ont pris place depuis la décennie 1980 jusqu'à nos jours, dans la plupart des pays industriels avan- cés, y compris au Canada et au Québec. Selon Lévesque, l'une des caractéristiques fondamentales de ces nouvelles politiques est la reconfiguration des rapports entre le social et l'économique : « Dans la configuration émergente, le social est non seulement un output, il est un input (partie prenante des avantages comparatifs) $»^{4}$. Dans ce sens, le social apparaît nettement comme un investissement et non plus uniquement comme une dépense. Cette approche s'accorde avec les pratiques des entreprises d'économie sociale qui relient l'économique et le social, développant des activités économiques afin de répondre à des besoins et à des aspirations sociales multiples.

Afin de compléter notre réflexion à ce stade-ci, ajoutons quelques mots pour rendre explicite la notion de territoire à laquelle nous nous référons lorsqu'il est question de développement local. Mobilisation des acteurs, démocratie économique et sociale, partenariat et réseaux, voilà un ensemble de termes qui nous renvoient à une conception territoriale axée sur le territoire entendu comme produit de l'action collective. Sans négliger que le territoire puisse être également le produit de l'action étatique, c'est sur le versant de l'action collective que nous nous attardons, cette définition du territoire étant plus propice à éclairer les convergences entre économie sociale, innovation et développement local.

\section{Des pratiques solidaires de développement : les CUMA au Québec}

Les CUMA sur lesquelles nous portons notre attention ont valeur d'exemplarité. Ces initiatives solidaires se retrouvent en milieu rural tout en refaisant le lien avec le mondial. Elles concernent des collectivités locales et des groupes confrontés à des difficultés socioéconomiques qui interviennent sur ces problèmes afin de dynamiser le développement de leurs collectivités.

\section{Contexte d'émergence des CUMA}

Comme nous l'avons déjà relevé en introduction, les Coopératives d'utilisation de matériel agricole sont un phénomène relativement récent au Québec, les premières étant apparues au début des années 1990. En 2004, on trouve 64 CUMA tant dans le Bas-SaintLaurent qu'en Abitibi, au Saguenay, en Beauce, dans les régions de Québec et Montréal, et une en Ontario ${ }^{5}$. Ces CUMA regroupent pas moins de 6400 producteurs-membres. Les immobilisations, en achat de ma- 
tériel agricole, totalisent 7 millions de dollars canadiens et plus de 120 machines et équipements sont partagés au travers de 560 branches d'activité en opération.

Pourquoi ce nouveau type de coopération au Québec ? Le contexte global dans lequel les producteurs agricoles ont évolué au cours des années 1970 et 1980 est révélateur à ce sujet : transformation de la profession d'agriculteur, celui-ci passant du statut de producteur familial à celui d'entrepreneur; entrepreneurs affiliés de plus en plus à des réseaux qui s'internationalisent. Il y eut le traité de libre-échange canadoaméricain, la globalisation des marchés, les nouvelles ententes du General Agreement on Tariffs and Trade (GATT) concernant notamment les échanges agricoles, la réduction des subventions publiques liées à l'agriculture. Voilà un environnement difficile pour les agriculteurs, qui plus est, étaient souvent fortement endettés. D'ailleurs, l'un des facteurs d'endettement le plus lourd est l'achat de machinerie agricole en propriété individuelle : en 1994, $23 \%$ des charges financières des agriculteurs, pris individuellement, allaient à l'achat d'équipement ${ }^{6}$. Pourquoi alors ne pas réduire ces coûts en se mettant ensemble afin d'acheter ce matériel ?

La réduction de l'endettement des agriculteurs est aussi un enjeu stratégique pour le développement de l'agriculture régionale et québécoise. Les entreprises agricoles dont la situation financière est précaire sont moins intéressantes à acheter pour la jeune relève. Tout cela est névralgique dans des régions où le nombre d'agriculteurs a déjà diminué depuis une trentaine d'années et où des terres sont abandonnées dans diverses municipalités. Les CUMA ne pouvaient-elles pas dès lors être un moyen de consolider l'agriculture locale, voire régionale, et contribuer ainsi à la dynamisation de plusieurs collectivités rurales?

Attardons-nous à un cas précis afin de mieux comprendre la dynamique de formation et de développement de ces coopératives. Avant la création de la première CUMA, il y a plusieurs contacts entre un noyau d'agriculteurs intéressés potentiellement par l'expérimentation et des agriculteurs membres de CUMA dans le Département du Tarn en France. Les producteurs québécois se rendent d'abord chez leurs « cousins » français et reçoivent ensuite la visite de ces derniers. Ceci leur permet de se familiariser avec le fonctionnement d'une CUMA et de bénéficier de l'expertise de leurs collègues français. À la suite de cette démarche de sensibilisation, la première CUMA québécoise voit le jour à l'hiver 1991 dans la région du Bas-Saint-Laurent, plus précisément dans une municipalité de la Municipalité régionale de comté Rimouski-Neigette. Une dizaine de producteurs sont sociétaires de cette nouvelle entreprise coopérative: neuf producteurs de lait et un éleveur de bœufs de boucherie. Ils ont entre 5 et 19 ans d'expérience sur leur propre ferme. Les objectifs fondamentaux que se donnent les sociétaires sont :

a) de se regrouper pour l'achat du matériel agricole afin de réduire les charges de mécanisation de chacun des producteurs sociétaires;

b) de développer des liens de convivialité qui assureront la communauté d'action en substituant aux préoccupations individualistes une nouvelle approche basée sur le respect, l'entraide, la solidarité et la confiance réciproque entre les producteurscoopérateurs.

D'emblée dans l'énonciation de ces objectifs, nous retrouvons la nature du problème d'endettement individuel auquel font face les agriculteurs, mais de plus leur volonté de s'allier pour faire face autrement, solidairement, à cette difficulté. Le caractère novateur de leurs pratiques commence déjà à prendre forme.

Cette CUMA connaît un développement considérable de ses activités et de son membership au cours des ans. À la fin de la décennie 1990, une quarantaine de producteurs sont sociétaires de la coopérative, ce qui correspond à la quasi-totalité des agriculteurs de la municipalité. La CUMA est propriétaire de 23 machines réparties dans 21 branches d'activités dont des semoirs à céréales, des machines à ensilage, des laveuses à pression et des tracteurs. La coopérative a contracté des emprunts pour plus de $300000 \$$ à la Caisse Desjardins locale, mais déjà $50 \%$ de cette somme est remboursée.

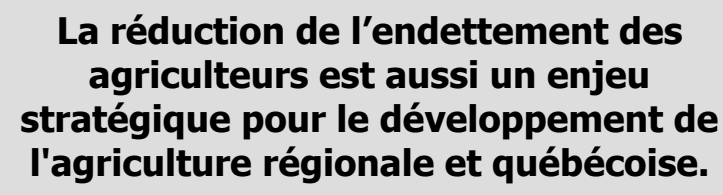

Examinons maintenant plus en détail les caractéristiques se dégageant des activités déployées par les cu- 
mistes ce qui nous permettra de faire le lien avec les principes et règles de fonctionnement des entreprises d'économie sociale, notamment coopératives, et avec l'innovation sociale.

\section{La structure des CUMA comprend trois niveaux d'organisation.}

\section{Entreprises au service de leurs membres et de leur collectivité}

La finalité de service des CUMA s'adresse d'abord à leurs membres. C'est pour répondre à un impérieux besoin économique vécu par les producteurs agricoles que les coopératives sont créées. Ces agriculteurs ressentent la nécessité de réduire leurs charges financières reliées à la mécanisation comme une condition de survie à long terme de leurs propres entreprises. Cet impératif nous renvoie à ce que Defourny nomme la condition de nécessité ${ }^{7}$. La coopération est « fille»de la nécessité, mais elle l'est également de la communauté de destin ressentie par les personnes qui deviendront membres. Cette communauté, ou encore ce sentiment d'appartenance à une condition commune, elle se construit au travers de l'action même des agriculteurs devenus sociétaires. Ceux-ci apprennent au sein de la CUMA à travailler autrement, de manière plus solidaire et conviviale. Ce n'est pas une mince affaire, pour des producteurs agricoles habitués à utiliser seuls leur matériel, que d'apprendre à organiser autrement leur travail sur leurs terres afin de tenir compte du temps dont chacun disposera pour l'usage du matériel mis en commun. Les agriculteurs doivent se préoccuper de ne pas dépasser le temps pour lequel ils ont loué l'équipement de la coopérative. Il faut éviter que l'utilisateur suivant ne soit pénalisé. De la même manière, le matériel doit être remis au suivant dans l'état où on l'a soi-même trouvé, c'est-à-dire impeccable. Des cumistes vont redécouvrir l'utilité des corvées pour faire les foins. La mise en œuvre de cette réorganisation du travail et la création de liens de confiance entre eux sont des caractéristiques des pratiques innovatrices des producteurs coopérateurs.

De plus, les CUMA ont un effet structurant important sur le développement socio-économique de leur collectivité locale. Mentionnons que, dans bien des cas, la majorité des producteurs locaux en sont membres. De plus, les charges financières de mécanisation des producteurs ont diminué fortement. On estime qu'un producteur cumiste dépense annuellement de $500 \$$ à $1000 \$$ pour de l'équipement loué à la coopérative, alors qu'auparavant il dépensait de $35000 \$$ à $40000 \$$ pour s'équiper! L'amélioration de la viabilité des entreprises agricoles est un gage d'avenir pour l'ensemble de la communauté locale dont l'agriculture est un vecteur essentiel de développement. Tout en améliorant la situation des fermes participantes à la coopérative, la mobilisation et l'implication des agriculteurs au sein de leur CUMA favorisent une meilleure cohésion sociale dans la communauté.

\section{Gouvernance interne et processus de décision démocratique}

Travailler autrement, c'est aussi apprendre à se gérer et à se diriger autrement. Relevons d'entrée de jeu l'intérêt du processus de démarrage d'une CUMA où une forte insistance est mise sur la sensibilisation et la formation des membres agriculteurs à la formule coopérative des CUMA, afin de s'assurer d'un noyau fondateur qui soit cohésif et conscient des réalités de l'apprentissage au fonctionnement de la gestion démocratique. Nous constatons que, dans la plupart des CUMA, prévaut une vitalité de participation. Quelques-unes ont connu des problèmes de gestion interne dus à un leadership autoritaire manifesté par certains membres ou encore au non-respect des règles encadrant la location du matériel agricole. Ces difficultés ont été affrontées par les coopérateurs concernés et réglées favorablement dans la plupart des cas. Seules quelques CUMA n'ont pas survécu aux étapes de démarrage et de consolidation.

La structure des CUMA comprend trois niveaux d'organisation. En commençant par la base, le niveau situé au plus près de l'organisation du travail quotidien des membres producteurs est celui des branches d'activités, plus ou moins nombreuses selon le type et le nombre de machines achetées par la coopérative. Viennent ensuite le conseil d'administration (CA) assisté d'un conseil exécutif $(\mathrm{CE})$ et l'assemblée générale des membres (AG).

Le travail relié au bon fonctionnement de la coopérative est accompli principalement par les membres bénévoles du $\mathrm{CA}$ et du $\mathrm{CE}$ ainsi que par chaque responsable de branche d'activité. Considérons, par exemple, le cas d'une branche liée à un semoir à céréales. Les membres adhérents de la branche signent un contrat d'engagement pour la location du semoir. 
Les adhérents peuvent se rencontrer au besoin, mais c'est la personne responsable de la branche qui voit au respect des règles de conduite de la CUMA, organise la planification du travail et la priorité d'utilisation du semoir en consultation avec les utilisateurs. Le responsable voit également à ce que le matériel soit entretenu, réparé et entreposé une fois terminée la saison agricole. Avant la tenue de l'assemblée générale annuelle de la coopérative, les membres de la branche se réunissent pour évaluer entre eux le travail accompli au cours de l'année. Ils examinent tout, autant l'évaluation du fonctionnement des machines, leur rendement, la qualité des rapports entre les membres usagers afin d'identifier et de corriger les difficultés rencontrées. Les membres de la branche d'activité remplissent une fiche complète d'évaluation, laquelle est acheminée au CA. Le conseil d'administration reçoit les rapports de chacune des branches d'activités et utilise cette information pour préparer la réunion de l'assemblée générale. La vitalité démocratique au sein de la grande majorité des CUMA est réelle et permet aux producteurs sociétaires de s'impliquer à divers niveaux de leur organisation. Ils sont associés de près à la prise de décision, tant par leur présence et leur droit de vote en assemblée générale que par le fonctionnement des branches d'activités et celui du conseil d'administration. Se gérer et se diriger de manière démocratique, en d'autres mots s'impliquer, voilà une autre caractéristique du caractère innovateur des CUMA.

\section{Solide autonomie de gestion}

Le financement des CUMA provient du capital social versé par les membres et d'emprunts auprès des Caisses Desjardins locales. Par exemple, dans chaque CUMA, les membres doivent souscrire ensemble un capital social équivalant à un minimum de $20 \%$ du coût d'achat du matériel agricole. Pour les $80 \%$ restants, la coopérative emprunte auprès de la Caisse locale. Celle-ci accepte d'autant plus facilement de financer que ces prêts sont garantis par la Financière agricole du Québec. En contrepartie, les producteurs membres s'engagent par contrat de location à utiliser le matériel acheté par la coopérative, ceci durant toute la durée du financement. Les CUMA peuvent ainsi respecter leurs engagements financiers auprès de leur Caisse.

Tout ceci illustre le développement d'une inter coopération entre les CUMA et les Caisses Desjardins, de même qu'un partenariat financier avec une institution publique.

Si cette inter coopération avec les Caisses Desjardins est maintenant acquise, ce ne fut pas le cas au début de l'expérimentation. Les premières CUMA ont eu de la difficulté à faire reconnaître le bien-fondé de leur nouvelle pratique. Les Caisses étaient alors réticentes à prêter face à des façons de faire nouvelles au Québec; elles craignaient d'investir dans ces nouvelles initiatives. Les membres fondateurs de la première CUMA, bénéficiant d'un capital social important dans leur communauté, ont convaincu les responsables de leur Caisse locale de courir le risque. Le précédent ayant été créé, et l'expérimentation de la CUMA s'avérant positive, les autres Caisses suivirent le mouvement, si je puis dire. À cet égard, l'appui financier du Mouvement Desjardins fut crucial. On peut toutefois regretter qu'il se soit fait tirer l'oreille au début de la mise en place des CUMA. En cela, Desjardins apparaît non pas comme un agent de changement luimême, mais plutôt comme un accompagnateur du changement ou de l'innovation promus par d'autres coopérateurs ou acteurs sociaux.

\section{Le financement des CUMA provient du capital social versé par les membres et d'emprunts auprès des Caisses Desjardins locales.}

Bref, les CUMA ne reçoivent pas de subsides publics. L'essentiel de leur financement provient du capitalsocial déboursé par les membres et de prêts consentis par les Caisses Desjardins locales. Les CUMA font ainsi la démonstration qu'elles sont capables de mobiliser l'épargne locale dans un projet de développement dont la propriété est également locale. Cette capacité de mobilisation de l'épargne est l'un des avantages identifiés par Pierre-Marcel Desjardins ${ }^{8}$ lorsqu'il parle de la coopérative comme outil de développement économique local et régional. C'est grâce à la connaissance qu'ils ont de leur milieu et à la confiance dont ils sont investis par les décideurs locaux que les cumistes sont aptes à canaliser l'épargne de leurs concitoyens. L'amélioration de la santé financière de chacun des producteurs-membres, ainsi que la faible dépendance des CUMA à l'égard de l'État, sont des objectifs centraux puisque ces coopératives ont été mises en place afin de réduire la vulnérabilité écono- 
mique, notamment, des agriculteurs dans un contexte de production mondialisée.

\section{En bref, des pratiques économiques solidaires}

Les cumistes poursuivent une double finalité économique et sociale : se regrouper pour l'achat de matériel agricole afin de réduire les charges de mécanisation de chacun des producteurs sociétaires; également, substituer aux préoccupations individualistes des producteurs indépendants une nouvelle approche basée sur le respect, l'entraide, la solidarité et la confiance réciproque entre producteurs-coopérateurs. Ici, l'économique et le social se renforcent mutuellement. Pour répondre à un besoin économique ressenti par tous, qui est celui de la réduction des charges financières dues à la mécanisation individualisée, les cumistes apprennent à se regrouper, à travailler autrement, à se faire confiance les uns les autres. Le social est bien un input, comme le souligne Lévesque, un levier essentiel pour le démarrage et la consolidation des CUMA.

\section{Pour les agriculteurs, la mise en place des CUMA leur permet de penser globalement, d'agir localement et solidairement.}

Divers témoignages reçus nous confirment la convivialité qui s'est mise en place dans la plupart des CUMA, le sens de la discipline et de la solidarité qui s'est développé au cours des années. Il y règne une organisation $\mathrm{du}$ travail efficace, une participation nombreuse aux assemblées, une rigueur dans l'entretien du matériel agricole et un respect des usagers de la coopérative. Les cumistes se sont dotés de regroupements ou réseaux régionaux où ils partagent leurs expériences et savoir-faire. Ils ont choisi pour le moment d'attendre avant de se regrouper en associations plus formelles.

\section{Conclusion}

Nous avons traité précédemment de pratiques de coopérateurs ayant implanté et développé avec ténacité de nouvelles organisations en milieu rural. Il s'agit des coopératives d'utilisation de matériel agricole qui, depuis leur apparition au début des années 1990, ont essaimé dans plusieurs régions du Québec. Elles se sont consolidées en tant qu'entreprises et s'inscrivent dans une démarche permettant la dynamisation des pratiques de développement local, dynamisation nécessaire à la consolidation des collectivités rurales face à un processus de mondialisation qui menace leur pérennité. Pour les agriculteurs, la mise en place des CUMA leur permet de penser globalement, d'agir localement et solidairement.

Tentons donc de synthétiser les acquis de leurs pratiques tant du point de vue de l'innovation sociale que du développement local. Concernant la forme et les motifs de leurs pratiques innovatrices, les cumistes ont recherché une solution à leur problème socio-économique qui se pose comme une solution inhabituelle, ou hors norme, dans le contexte québécois. Confrontés à un lourd endettement individuel, dû notamment à l'achat de machinerie agricole, les producteurs pionniers sont convaincus qu'il faut trouver à cela une solution collective débordant le cadre culturel des façons de faire «normales » des agriculteurs en la matière : le sens de la propriété individuelle. Acheter collectivement du matériel agricole, disons que ce n'était pas la pratique dominante parmi les agriculteurs. Qui plus est, en devenant propriétaire collectif de ce matériel, on ne peut plus travailler de la même façon qu'auparavant. Confiance réciproque entre les agriculteurs, discipline et responsabilité dans l'utilisation du matériel loué à la coopérative, établissement d'un horaire permettant à tous d'y trouver son compte, etc., voilà autant d'apprentissages que les cumistes ont réalisés pour le mieux-être de leurs fermes et de leurs entreprises coopératives. Il y a donc une innovation organisationnelle accompagnée, sinon précédée, d'une discontinuité par rapport à une approche culturelle de la propriété et de l'usage d'équipement fondée sur l'individualisme. Cette remise en cause de leur comportement est d'ailleurs énoncée clairement par les coopérateurs eux-mêmes. Les cumistes améliorent ainsi leurs conditions de vie en se mobilisant sans attendre que la solution à leurs difficultés ne vienne de l'extérieur.

Concernant le processus innovateur, il repose sur une large implication des protagonistes de la conception du projet à la consolidation des CUMA. Cette mobilisation s'étend de la prise de conscience du problème à l'exploration de solution en se tournant vers l'expérience française, à l'implantation des toutes premières coopératives et de celles qui suivront. Les producteurs sociétaires ont investi leurs CUMA en s'y impliquant à de multiples niveaux. On y retrouve une démocratie 
des usagers. En plus de ces usagers, l'innovation, pour s'implanter et se diffuser, a compté sur l'appui financier, notamment, de partenaires coopératifs tels les Caisses Desjardins, et public, telle la Financière agricole du Québec.

Les cumistes, par leurs pratiques, contribuent au développement local et territorial. Ils y contribuent par une meilleure occupation du territoire au travers les diverses activités humaines qu'ils y déploient. Ils permettent d'illustrer l'effet favorable d'une agriculture dynamique sur la consolidation des territoires ruraux. Cet effet structurant est bien démontré par Manuella Daniel dans une étude réalisée au Bas-Saint-Laurent auprès de deux coopératives agricoles importantes. Son étude démontre la contribution significative de ces entreprises associatives au développement de leurs collectivités par les emplois offerts, par la participation active de leurs membres ainsi que par leur esprit d'inter coopération' ${ }^{9}$.

Ils contribuent également à un développement s'articulant sur une synergie entre l'économique et le social. Les producteurs membres des CUMA participent à la reconstruction, ou à la consolidation, des liens sociaux dans leurs organisations, voire dans leur collectivité. N'oublions pas que, dans certaines municipalités rurales, la totalité des agriculteurs sont membres de la CUMA locale. Ils assurent ainsi une plus grande cohésion sociale de leur milieu, gage d'un développement économique mieux assuré et enraciné sur son territoire. Le renouvellement des liens sociaux est important lorsque nous parlons d'innovation sociale et de satisfaction des besoins humains. En effet, l'innovation favorise l'inclusion sociale des individus et des acteurs concernés ${ }^{10}$.

En terminant, les cumistes participent à une mobilisation importante de ressources humaines et financières tant par le nombre de membres impliqués dans les CUMA que par les ressources financières mises en œuvre. Ils contribuent de même à un partenariat entre divers acteurs sociaux de la société civile.

\section{Notes et références}

1 Demoustier, D. (2001). L'économie sociale et solidaire, Paris, La Découverte et Syros.

2 Chantier de l'économie sociale (2001). De nouveau, nous osons, document de positionnement stratégique, Montréal, p. 28.

3 Defourny, J. et J.L. Monzon Campos (1992). Économie sociale. Entre l'économie capitaliste et l'économie publique, Bruxelles, De Boeck Université, p. 230.

4 Lévesque, B. (2001). Le développement régional et local, avant et après la mondialisation, Cahiers du CRISES, $n^{\circ}$ ET0116, CRISES, Montréal, juin, p. 14.

5 Morneau, C. (2004). Mise à jour des données sur les CUMA, document à usage interne, ministère de l'Agriculture, des Pêches et de l'Agroalimentaire du Québec, Rimouski; Morneau, C. (1999). «CUMA : une structure économique », communication présentée à l'Université rurale québécoise au Bas-Saint-Laurent, Trois-Pistoles, octobre.

6 Morneau, C. (1994). La coopérative d'utilisation de matériel agricole (CUMA). Un outil de formation et de développement au service de l'agriculture régionale : du concept à l'expérimentation, mémoire de Maîtrise en développement régional, Université du Québec à Rimouski.

7 Defourny, J. (1995). «L'avenir des pratiques coopératives dans un monde en mutation », dans M.-T. Seguin (dir.), Pratiques coopératives et mutations sociales, Paris, L'Harmattan, p. 13-25.

8 Desjardins, P.-M. (1995). « Le coopératisme, outil de développement économique régional ? », dans M.-T. Seguin, Pratiques coopératives et mutations sociales, Paris, L'Harmattan, p. 113-122.

9 Daniel, M. (2004). «Les coopératives agricoles et leurs impacts sur le développement régional : étude comparative entre la France et le Québec », mémoire de Maîtrise en développement régional, UQAR.

10 Hillier, J., F. Moulaert et J. Nussbaumer (2004). « Trois essais sur le rôle de l'innovation sociale dans le développement territorial », Géographie, Économie et Société, vol. 6, $\mathrm{n}^{\mathrm{o}} 2$, avril-juin, p. 129-152. 